\title{
Land Mine Detection With Neutron Back Scattering Imaging Using a Neutron Generator
}

\author{
Victor Bom, Mostafa A. Ali, and Carel W. E. van Eijk
}

\begin{abstract}
The neutron back scattering technique may be used to search for low-metallic land mines. An advantage of this technique is the speed of detection: the scanning speed may be made comparable to that of a metal detector. The method is sensitive to soil moisture. A limitation of the method is therefore that the soil must be sufficiently dry. The neutrons are produced with a pulsed neutron generator. An image of the back scattered thermal neutron radiation is obtained with a two dimensional position sensitive detector. Getting optimal settings for the detector system is described. The mine detection time as function of the neutron pulse parameters is investigated. Results with various dummy mines are presented.
\end{abstract}

Index Terms-Land mine detection, neutron generator, neutron scattering.

\section{INTRODUCTION}

$\mathbf{L}$ ANDMINE detection with neutrons is based either on prompt $(\mathrm{n}, \gamma)$ reaction or on neutron scattering on hydrogen. With the first method the elemental composition of the irradiated volume is determined. It is a slow method and therefore not very well suited for scanning a surface for mines. The second method, called neutron backscattering (NBS) [1]-[5] relies on the thermalization of fast neutrons. In this technique a thermal neutron detector monitors slow neutrons which emerge form the soil while irradiating the soil volume under investigation with fast neutrons. Above a hydrogen-rich anomaly e.g., a land mine, the thermal neutron flux increases, because hydrogen is an extremely efficient moderator. Since land mines have a much higher concentration of hydrogen atoms than a normal dry sandy soil, the NBS method may be used to detect mines in such a soil.

Since 1998, different detector system have been developed and tested [1], [6]. They have demonstrated the feasibility of the NBS method. It has become apparent that the NBS method is one of the fastest ways to find land mines. Detection times are comparable with those of the well known "metal detector". For this reason the emphasis has been on the development of hand held systems and on the combination of a NBS detector with a metal detector. The main limitation of the NBS method

Manuscript received November 17, 2004; revised June 7, 2005. This work was supported by the Dutch Ministry of Defense.

V. Bom, and C. W. E. van Eijk are with the Delft University of Technology, Delft 2629 JB, The Netherlands (e-mail: vb@iri.tudelft.nl).

Mostafa A. Ali is with the Atomic Energy Authority, Nuclear Research Center, Reactor and Neutron Physics Dept., Abu Zabal, Cairo, Egypt. and is supported by an IAEA Grant.

Digital Object Identifier 10.1109/TNS.2006.869841 lies in the soil humidity. Around $12 \%$ mass humidity(weight fraction of water), the concentration of hydrogen in the soil becomes equal to the concentration of hydrogen in the land mine. This results in a loss of contrast between the mine and the surrounding soil. The application of the NBS technique, on which the DUNBID detector is based, is therefore limited to relatively dry soils such as occur in arid climates with desert surroundings. Countries around the Sahara desert and in the middle east (Israel, Lebanon, Afghanistan) are likely candidates. We estimate that about half of all 50 million mines (of which 16 million in the Egyptian desert alone) are placed in soils dry enough for a NBS device to be useful.

While the NBS method can be demonstrated with a single slow neutron detector, the sensitivity for mine detection is limited to large mines at shallow depths. The present work aims at considerably increasing the sensitivity for mine detection The sensitivity is optimized in the following ways. Firstly, by obtaining an "image" of the backscattered radiation instead of just counting the number of slow neutrons, a hydrogen-rich area may be found more easily. Because at the mine location the mine/no-mine signal ratio will be higher than for the image as a whole, the mine detection probability increases.

Secondly, an improvement may be achieved by using the neutron time-of-flight technique. Applying a time window on the neutron time-of-flight, the no-mine response may be decreased while leaving the on-mine response unharmed. This improves the "signal-to-noise" ratio. This however requires a pulsed neutron generator instead of a radioactive source.

Tagged neutron sources, where the direction of the emitted neutron is known, can also provide a significant reduction of the mine/no-mine signal ratio. However the maximum count rate with such sources is very limited because only one neutron can be allowed in the setup at any time, and the neutrons may have life times into the millisecond range.

The above considerations have led us to investigate into the applicability of an imaging device with a large detection plane close to the soil [7]. An imaging system will have to sit on a cart or on a vehicle, because of the weight of the source-detector system and because of the considerable amount of electronics needed. Such a system could be applied in front of a vehicle to detect large anti-tank mines at a relatively high-speed. The vehicle could also carry other systems to improve the overall detection capability. The obtained image would be well suited for data fusion with other imaging devices [8] like Ground Penetrating Radar systems [9], [10], or Schiebel arrays [11]. 


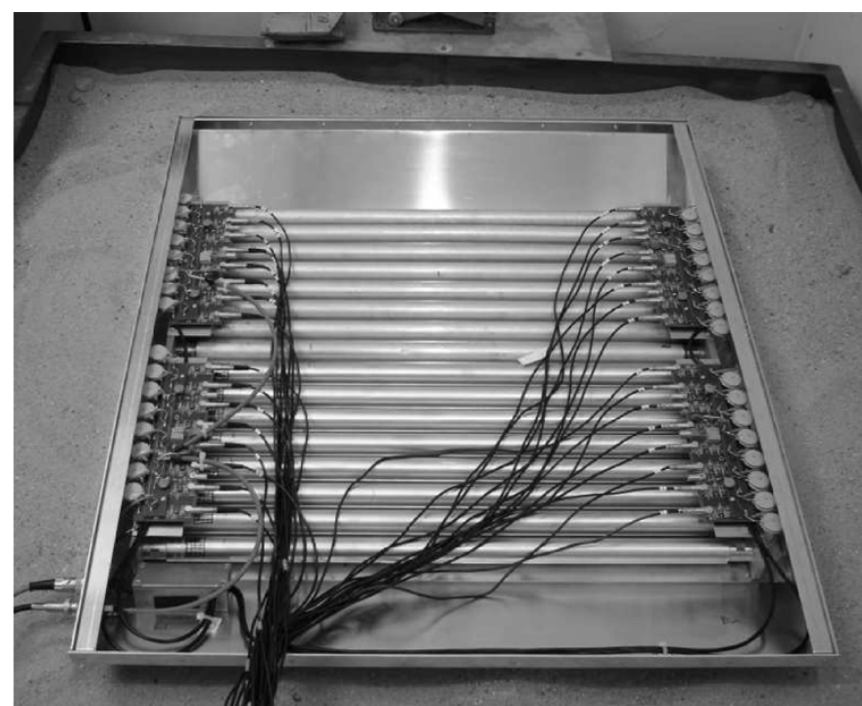

Fig. 1. DUNBID detector. $16{ }^{3} \mathrm{He}$ tubes are mounted inside a tray. On the left and right, the four preamplifier boards are shown, with the HV coupling capacitors just visible. Thirty-two cables feed the amplified signals into a position calculation unit (not shown).

\section{EXPERIMENTAL SETUP}

\section{A. Detector}

DUNBID, the Delft University Backscattering Imaging Detector, shown in Fig. 1, consists of sixteen position sensitive thermal neutron ${ }^{3} \mathrm{He}$ proportional counter tubes placed in a tray (Aluminum tubes, length: $50 \mathrm{~cm}$, diameter: $2.54 \mathrm{~cm}$, pressure: 10 bar, tube pitch: $34 \mathrm{~mm}$ ). The position of a neutron hit along the tube is determined by charge devision. The preamplifier's and associated position calculation electronics were obtained from the Rutherford Appleton laboratory.

The tube number and digital position of a neutron hit are fed into a process computer via an interface. A gate can be applied to this interface to set a time window. The computer operates stand-alone, and is controlled via the Internet. The dead time of the system is around $5 \mu \mathrm{s}$, which is mainly due to interrupt handling by the computer.

The spatial resolution in the detector plane is, in the direction perpendicular to the tubes of course equal to the tube pitch (34 $\mathrm{mm}$ ), and in the direction parallel to the tubes equal to the tube length divided by the number of bins, so $500 / 16=31 \mathrm{~mm}$. This resolution is sufficient to "see" all sizes of land mines. The mine image as measured by the detector does not reflect the actual mine shape or size, but is always circular with a diameter of around $15 \mathrm{~cm}$ (see [6]). This is explained by the large distance a thermalized neutron can drift underground before emerging at the surface. Smaller mines produce a spot with an only slightly smaller diameter, but the mine depth and the distance of the detector from the ground surface (standoff distance) also influence the shape of the image.

The detector efficiency is almost $100 \%$ for neutrons with thermal energies. At $2.5 \mathrm{MeV}$ the efficiency is around $10^{-4}$. This means that still some high energy neutrons from the source can be detected. If the number of such neutrons is high compared to the number of thermal neutrons there will be a noticeable detector response, which could make a mine signal difficult to recognize. This can occur if the neutron source is close to the detector tubes (see also Section III-A-1).

Because the source is relatively close to the soil surface, there will be a variation in sensitivity over the detector area. Close to the edge the flux of fast neutrons will be markedly lower than in the center. A mine placed in the central region will have a better chance of being spotted compared to a mine at the detectors edge.

\section{B. Test Site}

Indoor measurements were done on very dry sand with a humidity below $0.5 \%$ (mass). The specifications of the dummy mines we used are listed in Table I.

\section{Neutron Generator}

Pulses of neutrons were made with a GENIE 16C DD neutron generator The neutrons are mono-energetic with an energy of $2.45 \mathrm{MeV}$ to avoid electrical interference from the generator and its cables on the preamplifiers or on the ${ }^{3} \mathrm{He}$ tubes, the tray, holding the detectors, was closed with a metal lid. The generator was laid down on top of this lid. The point of neutron emission is then about $10 \mathrm{~cm}$ above the bottom of the tray. The generator was used at an emission rate of about $6.6 \cdot 10^{5} \mathrm{n} / \mathrm{s}$, resulting in a total count rate in the detector of about $3500 \mathrm{c} / \mathrm{s}$.

\section{OPTIMIZATION}

The performance of the detection system is determined by the values of a set of parameters such as:

- the neutron pulse characteristics: width and repetition rate;

- the time-of-flight window: delay and width;

- the mine characteristics: size, depth;

- the soil characteristics: humidity.

To get a handle on the quality of a set of parameters the entity $t_{3 \sigma}$ is introduced. $t_{3 \sigma}$ is the measurement time needed to get an mine signal which is three standard deviations above the signal without a mine. This relates to a mine detection efficiency of $99.7 \%$. The smaller the $t_{3 \sigma}$ value, the higher the mine detection sensitivity. Basic counting statistics arguments lead to

$$
t_{3 \sigma}=\frac{1}{B} \frac{f+1}{(f-1)^{2}}
$$

where $B$ is the count rate without a mine in a certain detector area, and $f$ is the ratio of the mine/no-mine count rates in that area. So, a high sensitivity may not only be obtained with a high mine/no-mine signal ratio, but also with a high count rate.

\section{A. Time-of-Flight Window}

The neutron time-of-flight is the time between the emission of the neutron and the absorption in the detector. There is an optimum setting for the window on the neutron time-of-flight. Short times are related to fast neutrons which come straight from the source or which are not properly thermalized. Long flight times are due to neutrons which have been scattering around through the surroundings over a large distance. Both such neutrons carry little information regarding the mine. A properly set time window will reduce the contribution of these neutrons and will thus decrease the no-mine signal. 
TABLE I

SPECIFICATIONS OF THE DUMMY MINES

\begin{tabular}{rccl}
\hline mine & size $(\mathrm{cm} \times \mathrm{cm})$ & No. of H-atoms per mine & Description \\
\hline AT & $\varnothing 20 \times 4$ & $4.5 .10^{25}$ & nylon casing filled with melamine \\
DLM2.2 & $\varnothing 8 \times 3.4$ & $6.4 .10^{24}$ & see [12] \\
\hline
\end{tabular}

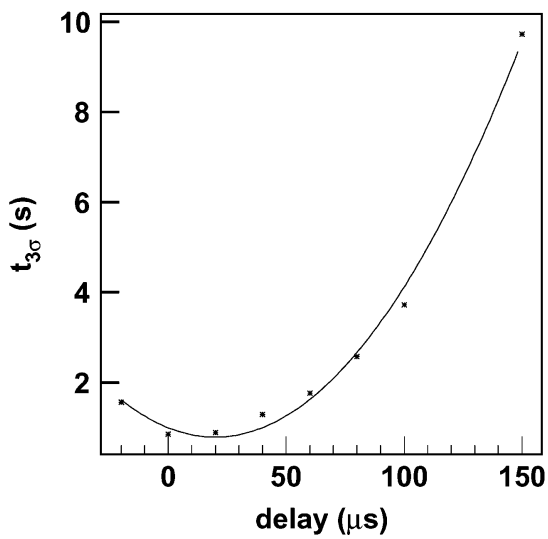

Fig. 2. $t_{3 \sigma}$ as function of the time-of-flight window delay. The window width is fixed at $20 \mu \mathrm{s}$. AT dummy mine at $6 \mathrm{~cm}$ depth. Measurement time per point: $300 \mathrm{~s}$, neutron pulse width $50 \mu \mathrm{s}, 1 \mathrm{kHz}$ repetition rate, emission $6.6 .10^{5} \mathrm{n} / \mathrm{s}$. The curve just guides the eye.

The time-of-flight window is characterized by a delay and a width. The delay is defined with respect to the end of the neutron pulse. Due to the width of the neutron pulse itself the effective time-of-flight window is not equal to the gate as applied electronically to the detector output pulses. Instead, for the effective window it follows:

and

$$
t_{\text {delay }}^{\text {effective }}=t_{\text {delay }}^{\text {gate }}
$$

$$
t_{\text {width }}^{\text {effective }}=t_{\text {width }}^{\text {gate }}+t_{\text {width }}^{\text {neutron pulse }} \text {. }
$$

1) Delay: For negative values of the delay the time-of-flight window overlaps with the pulse of fast neutrons from the generator. These fast neutrons cause a peak in the image spectra at the source position. Therefore a small positive delay is necessary. A too large delay setting however will be unfavorable because of a lower count rate and a lower mine/no-mine ratio. It is assumed that the delay value will be independent of the setting of the other parameters. To determine the optimum delay setting, the time-of-flight window delay was varied, keeping the window width constant. The results are shown in Fig. 2.

We choose a value of $10 \mu$ s for the time-of-flight window delay. It was used in all following measurements.

2) Width: Using the $10 \mu$ s delay, the width of the time-offlight window was varied. The results are shown in Fig. 3. It shows a broad minimum between 150-200 $\mu$ s.

For small widths, the $t_{3 \sigma}$ value is high because of the low count rate. For greater widths an increasing number of long lived neutrons come into the window, deteriorating the mine/no-mine signal ratio $f$. This effect is outweighed by the increasing count rate up to around $200 \mu \mathrm{s}$.

\section{B. Neutron Generator}

The pulse repetition rate was varied between 0.33 and 3.33 $\mathrm{kHz}$. The neutron generator allows for a minimum pulse width

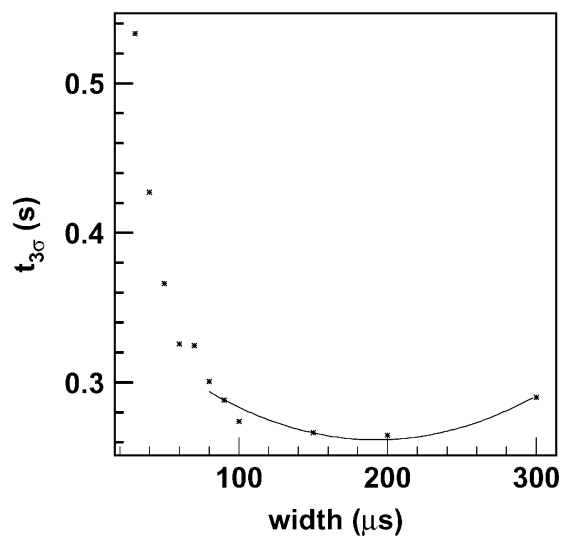

Fig. 3. $t_{3 \sigma}$ as function of the time-of-flight window width. The window delay is fixed at $10 \mu \mathrm{s}$. AT dummy mine at $6 \mathrm{~cm}$ depth. Measurement time per point: $300 \mathrm{~s}$, neutron pulse width $50 \mu \mathrm{s}, 1 \mathrm{kHz}$ repetition rate, emission $6.6 .10^{5} \mathrm{n} / \mathrm{s}$. The curve guides the eye.

TABLE II

INFLUENCE OF THE NEUTRON GENERATOR REPETITION RATE. AT DumMY Mine AT $6 \mathrm{~cm}$ DePTH. $t_{3 \sigma}$ VALUES FOR SOME GENERATOR AND DELAY WINDOW SETTINGS

\begin{tabular}{ccccc}
\hline pulse rate $(\mathrm{kHz})$ & $t_{\text {width }}^{\mathrm{n} \text {-pulse }}(\mu \mathrm{s})$ & $t_{\text {width }}^{\text {gate }}(\mu \mathrm{s})$ & $t_{\text {width }}^{\text {effective }}(\mu \mathrm{s})$ & $t_{3 \sigma}(\mathrm{s})$ \\
\hline 0.333 & 150 & 10 & $10-170$ & 2.9 \\
1 & 150 & 10 & $10-170$ & 2.3 \\
1 & 50 & 110 & $10-170$ & 0.31 \\
2 & 25 & 110 & $10-145$ & 0.27 \\
3.33 & 15 & 110 & $10-135$ & 0.26 \\
3.33 & 15 & 75 & $10-100$ & 0.31 \\
\hline
\end{tabular}

of $5 \%$ of the repetition frequency. So the corresponding minimum pulse widths are between 150 and $15 \mu \mathrm{s}$.

Higher frequencies allow for shorter settings of the neutron pulse width, and thus for a more narrow effective time window. This may improve the mine/no-mine ratio, as not mine-related neutrons are filtered out better. At lower frequencies there will be less neutrons left over from the previous pulses in the time-offlight window, but then the minimum neutron pulse width, allowed by the generator, is larger, with a correspondingly wider effective time window. $t_{3 \sigma}$ values for some settings are reported in Table II for the AT dummy mine buried at $6 \mathrm{~cm}$ depth.

Table II shows that higher frequencies are somewhat more favorable. The effective time-of-flight window can be set by a small neutron pulse width combined with a wide gate (the four last table entries), or by a large neutron pulse width combined with a narrow gate (first two table entries). The count rate in the latter case is an order of magnitude lower compared to the rate in the first case, explaining the larger $t_{3 \sigma}$ values.

\section{MEASUREMENTS}

The detector tray was placed on the sand surface, so with a zero standoff distance. This relates to applications where the 

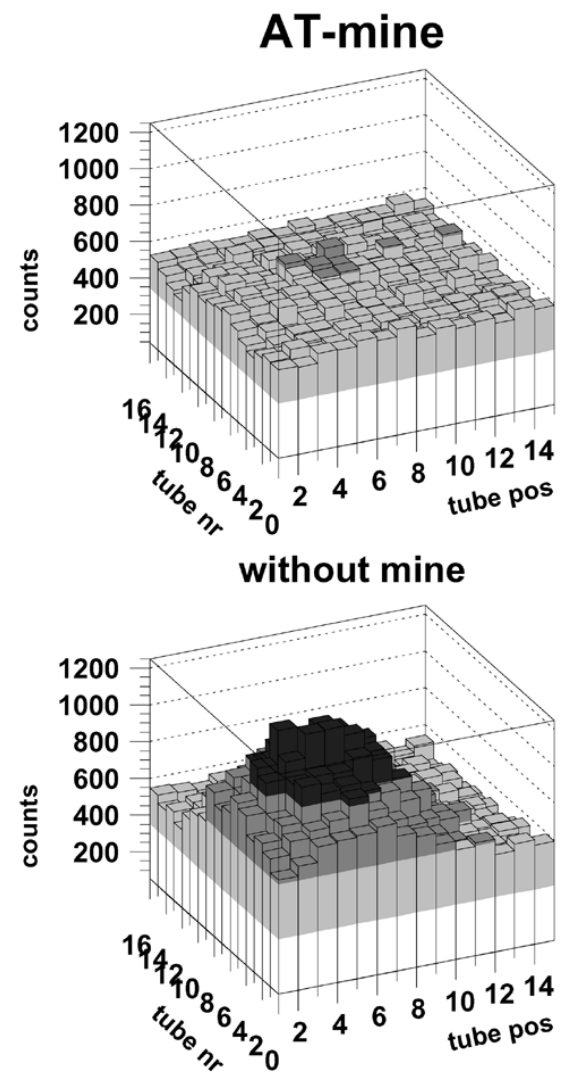

with mine

Fig. 4. Measurement with the neutron generator of the AT dummy mine at a depth of $8 \mathrm{~cm}$. Neutron generator: $6.6 .10^{5} \mathrm{n} / \mathrm{s}, 50 \mu$ s neutron pulse width, $1 \mathrm{kHz}$ repetition rate. Measurement time: $300 \mathrm{~s}$. Accepted times-of-flight: $10-110 \mu \mathrm{s}$. Top: measurement without the mine, bottom: result with the AT-mine.

surface is tread safe, for instance after a mechanical detonation treatment ("steel wheels", "flails", etc.). The tray can than be dragged behind a mine protected vehicle to find mine remnants, or unexploded mines. The mine depth is the distance from the soil surface to the top of the mine.

The experimental setup is situated inside an earth covered "bunker": an underground bombproof shelter. The heavy walls and the earth cover cause a considerable slow neutron background, which is uncorrelated to the presence of the mine. The experimental setup is situated in a corner of the bunker. The sides defined by "tube number 15" and "tube position 15" are closest to the walls; the corner being at $(15,15)$ As a result their is a general rate increase going from position $(0,0)$ to position $(15,15)$. Because of the distance between the detector and the walls these background neutrons appear predominantly at later time window settings.

\section{A. Anti Tank Mine}

The measurement result for the AT dummy mine is shown in Fig. 4. The no-mine response is nearly flat. For the mine, the $t_{3 \sigma}$ value is $0.3 \mathrm{~s}$.

Effects of the background neutrons are minimal because of the early timing window setting.

\section{B. Anti Personnel Mine}

The response of the DLM2 mine buried at $10 \mathrm{~cm}$ depth is shown in Fig. 5. The figure shows the peak in the center due to

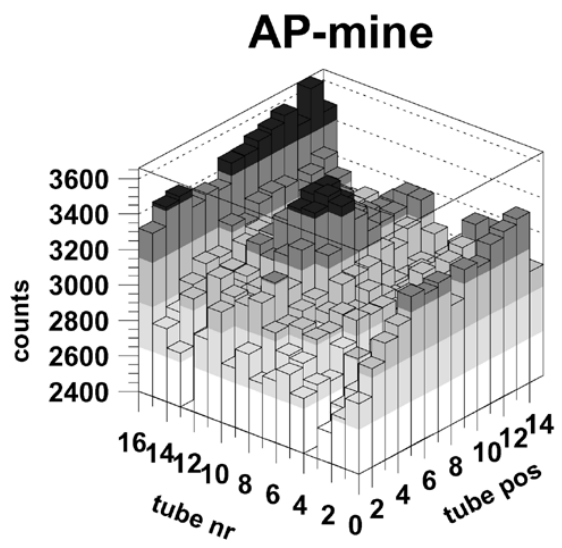

Fig. 5. Measurement with the neutron generator of the DLM2 dummy mines at a depth of $10 \mathrm{~cm}$. Neutron generator: $6.6 .10^{5} \mathrm{n} / \mathrm{s}, 20 \mu$ s neutron pulse width, $2.5 \mathrm{kHz}$ repetition rate. Measurement time: $300 \mathrm{~s}$. Accepted times-of-flight: $10-230 \mu \mathrm{s}$.

the mine. The fourth tube is malfunctioning resulting in a too high rate at higher positions. The observed $t_{3 \sigma}$ value is $6.9 \mathrm{~s}$.

Effects of the background neutrons are more clearly seen as compared to Fig. 4 since a later timing window setting is used. The high count rate in the first and the last tube is caused by slow background neutrons which are entering from the side into these two tubes. The other tubes all have tubes alongside them which is why they don't suffer from this effect. The tube ends of course also experience a raised neutron flux. But this raise is not detected because the outermost $2 \mathrm{~cm}$ at the tube ends are insensitive.

This means that with a neutron generator giving some $10^{8} \mathrm{n} / \mathrm{s}$ the DLM detection time would be reduced to below $0.1 \mathrm{~s}$ !

\section{Standoff Distance}

The response of the detector without a mine is flat. The detection of the mine is based on the recognition of a wide peak on this flat background. This concept does not depend upon the actual standoff distance. This is in contrast with detectors which rely on an increase of the overall count rate for the detection of a mine.

\section{CONCLUSION}

The new thermal neutron back scatter device easily detects mines quickly. With realistic source strengths, sub-second detection times seem possible. This is based on simple counting statistics. Application of more sophisticated data analysis methods where the peak shape is properly taken into account will increase the sensitivity. These short detection times in combination with the detector size of $50 \times 50 \mathrm{~cm}$ will allow for scanning speeds of a few kilometers per hour.

The maximum depth at which a mine can be found depends on many factors, the most important ones being the allowed measurement time and the soil moisture. In a dry homogeneous sand soil it appears that anti-tank mines can be found within 1 second to a depth of at least $20 \mathrm{~cm}$. For anti-personnel mines this maximum depth is $15 \mathrm{~cm}$. 


\section{REFERENCES}

[1] C. Datema, V. R. Bom, and C. W. E. van Eijk, "Land mine detection with the neutron back scattering method," IEEE Trans. Nucl. Sci., vol. 48, no. 4, pp. 1087-1091, Aug. 2001.

[2] F. D. Brooks, A. Buffler, and S. M. Allie, "Detection of plastic land mines by neutron back scattering," in 6th International Conference on Applications of Neutron Science, Crete, Greece, Jun. 1999.

[3] C. Datema, V. R. Bom, and C. W. E. van Eijk. DUNBLAD, the delft university neutron backscattering landmine detector. presented at Proceedings of the Fifth International Symposium on Technology and the Mine Problem. [Online]. Available: http://www.demine.org/symposia/5th-NPS-2002/PAPERS/PARALLEL/NUCLEAR/DATEMA.PDF

[4] G. Nebbia et al.. DIAMINE (detection and imaging of antipersonnel landmines by neutron backscattering tewchnique). presented at Proceedings of the Fifth International Symposium on Technology and the Mine Problem. [Online]. Available: http://www.demine.org/symposia/5th-NPS-2002/PAPERS/PARALLEL/NUCLEAR/NEBBIS.PDF

[5] J. Csikai, B. Király, and R. Dóczi, "Application of neutrons to plastic landmines detection," in IAEA/PS/RC-799-2, Vienna, Austria, Sep. 2001, pp. 46-54. cD available from IAEA.

[6] C. Datema, V. R. Bom, and C. W. E. van Eijk, "Optimization of the delft university neutron back scattering land mine detector (DUNBLAD)," in 7th International Conference on Applications of Neutron Techniques, Crete, Greece, Jun. 2001.
[7] V. R. Bom, C. W. van Eijk, and M. A. Ali, "DUNBID, the Delft university neutron backscattering imaging detector," in Appl. Rad. and Isot., 8th International Conference on Applications of Nuclear Techniques, Nov./Dec. 2005, pp. 559-563.

[8] "Demonstration of Multi-Sensor Landmine Detection Techniques," DEMAND, final report, http://www.eudem.vub.ac.be/files/demand-final_report.pdf, Jun., 2004. The DEMAND consortium, EC Funded Project: IST-200-25 351.

[9] M. Bradley, T. Written, M. Duncan, and B. McCummins, "Mine detection with a forward-looking ground-penetrating synthetic aperture radar," in Detection and Remediation Technologies for Mines and Minelike Targets VIII, R. Harmon, J. J. H. Holoway, and J. Broach, Eds. Orlando, Florida, USA: SPIE-The International Society of Optical Engeneering, Apr. 2003, vol. 5089, pp. 334-347.

[10] D. M. Port, P. Gardiner, and K. Long, "Analysis and application of a vehicle mounted ground penetrating radar array," in Detection and Remediation Technologies for Mines and Minelike Targets VIII, R. Harmon, J. J. H. Holoway, and J. Broach, Eds. Orlando, FL: SPIE-The International Society of Optical Engeneering, Apr. 2003, vol. 5089, pp. 348-357.

[11] VAMIDS: The Vehicular Array Mine Detection System (2004). [Online]. Available: www.schiebel.net/pages/vamid-feat.html

[12] F. D. Brooks, M. Drogs, A. Buffler, and S. M. Allie, "Detection of antipersonnel land mines by neutron scattering and attenuation," Appl. Rad. and Isot., vol. 61, pp. 27-34, Jul. 2004. 